\title{
The role of imbalance between urinary hexanoyl-lysine and total antioxidant capacity levels and its relation to plasma superoxide dismutase levels in autism spectrum disorder
}

\author{
Kunio Yui ${ }^{1 *}$, Hitomi Sasaki ${ }^{1}$ and Nasoyuki Tanuma ${ }^{2}$ \\ ${ }^{1}$ Department of Urology, Fujita Health University, Toyoake, Aichi, Japan \\ ${ }^{2}$ Department of Pediatrics, Tokyo Metropolitan Fuchu Medical Center for the Disabled, Tokyo, Japan
}

\begin{abstract}
Introduction: The imbalance between oxidants and antioxidants contributes to the pathophysiology of ASD.

Objective: First, we evaluated the link between the oxidative stress related biomarkers and social impairment of autism spectrum disorder (ASD).

Methods: We measured the urinary levels of an oxidative stress biomarker, hexanoyl-lysine (HEL), the total antioxidant capacity (TAC) and the DNA methylation biomarker 8-hydroxy-2'-deoxyguanosine (8-OHdG), and the plasma levels of a major antioxidant enzyme, superoxide dismutase (SOD). We examined the relationship between these four biomarkers and social responsiveness in 20 individuals with ASD and in 11 healthy controls. The sex and age distributions (11.4 \pm 5.1 years vs. 13.9 \pm 6.6 years) were not significantly different between the groups. Social responsiveness was assessed using the Social Responsiveness Scale (SRS).
\end{abstract}

Results: Dietary TAC from chocolate, biscuits and cookies, jam and marmalade were significantly higher in the ASD group than in the control group. Urinary TAC levels were significantly lower, but the urinary HEL levels were significantly higher in the ASD group compared to the control group. Urinary 8-OHdG levels and plasma SOD levels were not significantly difference between the groups. SRS scores were significantly higher in the ASD group than in the control group. Stepwise regression analysis revealed that urinary TAC and HEL levels may predict differences in the biomarkers and SRS scores between the groups.

Conclusion: A critical imbalance between the urinary HEL and TAC levels may contribute to impaired social responsiveness in individuals with ASD without the DNA methylation.

\section{Introduction}

The prevalence of autism spectrum disorders (ASD) has markedly increased in recent years [1]. ASD symptoms usually appear during early development and generally lead to serious lifelong limitations in function; thus, both genetic and environmental factors may contribute to its development $[2,3]$. The shift in the balance between oxidants and antioxidants in favor of oxidants is termed "oxidative stress" [4]. Regulation of reduction/oxidation (redox) state is important for cell viability, proliferation and organ function [4], however, imbalance of oxidant/antioxidant balance such as overproduction of ROS became toxic to neurons, inducing DNA methylation [5] and various tissues damage [6]. Because the brain is highly vulnerable to oxidative stress during early development [6], oxidative stress-induced neuronal damage may occur in genetically predisposed individuals [7]. Thus, deficits in antioxidant capacity may important in the etiology of ASD. The total antioxidant capacity (TAC) in urine and plasma is a parameter used to characterize the antioxidant status of the body $[8,9]$.

Recent studies in ASD have shown significantly reduced urinary levels of TAC, indicating increased vulnerability to oxidative damage [6], and significantly higher urinary levels of 8-hydroxy2 -deoxyguanosine $(8-\mathrm{OHdG})$ [10]. further, the urinary levels of oxidative stress markers such as hexanoyl-lysine (HEL) were found to be elevated in children with ASD, and higher HEL values correlated with the hyperactivity component of the Childhood Autism Rating
Scale [11]. Thus, urinary oxidative stress-related biomarkers, including HEL, which is reportedly a more sensitive biomarker of oxidative stress [12], TAC and biomarkers of DNA methylation may provide useful information about oxidative stress-related brain damage. However, few studies have measured urinary levels of HEL, TAP and 8-OHdG.

Increased ROS with a concomitant decrease in antioxidant capacity may induce more serious damage in diseases such as leukemia $[13,14]$. However, few reliable studies have shown increased HEL to be concomitant with decreased antioxidant capacity in ASD.

Interestingly, a recent study revealed that decreased serum levels of SODs, which are major antioxidant enzymes [15] and contribute to

Correspondence to: Kunio Yui MD PhD, Visiting Professor, Department of Urology, Fujita Health University, Toyoake, Aichi, Japan, Tel: 8178791 8981; Fax:81 79237 8755; E-mail: yui16@bell.ocn.ne.jp

Key words: urinary total antioxidant power, hexanoyl-lysine (HEL), 8-hydroxy-2'deoxyguanosine (8-OHdG), social responsiveness scale, autism spectrum disorder, dietary antioxidant power, urinary total antioxidant capacity levels, urinary HEL levels, urinary 8-OHdG levels, imbalance between oxidants, antioxidants Superoxide dismutase, autism spectrum disorders

Received: February 02, 2018; Accepted: February 14, 2018; Published: February 19,2018 
Yui K (2018) The role of imbalance between urinary hexanoyl-lysine and total antioxidant capacity levels and its relation to plasma superoxide dismutase levels in autism spectrum disorder

human neurodegenerative disorders [16], may also be implicated in the pathophysiology of ASD [17]. Previous studies have also reported that altered plasma levels of enzymatic antioxidants such as SOD, ceruloplasmin $(\mathrm{Cp})$, and transferrin (Tf) play an important role in ASD [18-25]. In spite of the important pathophysiological contribution of SOD in ASD, the role of the plasma SOD levels in relation to the urinary levels of oxidative stress-related biomarkers remains unclear.

These considerations taken together, we pursued three avenues of research in this work. First, we evaluated the link between the urinary levels of HEL, TAC and 8-OHdG and social responsiveness in individuals with ASD. Next, we assessed the role of plasma SOD levels in relation to these urinary biomarkers. Finally, we determined the relationship between HEL and TAC in urine of individuals with ASD. Because previous clinical studies have found that urinary levels of TAC are affected by diet $[26,27]$, we also assessed dietary nutrient intake and estimated dietary TAP.

\section{Subjects and methods}

\section{Subjects}

This study included a total of 31 young, physically healthy individuals in Hanshin area (Kobe and Osaka prefectures) participated in this study. They were recruited from the medical care facilities of the Research Institute of Pervasive Developmental Disorders of Ashiya University based on their presenting for a medical consultation between October 2013 and September2015. ASD was diagnosed based on the DSM-5 criteria [28] and was confirmed by the Autism Diagnostic Interview-Revised (ADI-R) [29]. Two psychiatrists specializing in ASD and developmental disorders diagnosed all 20 individuals with ASD. Among the 31 participants, 20 had an independent clinical diagnosis of ASD (13 males and 7 females, mean age: $11.1 \pm 5.2$ years old, age range: 6-22 years old), and the remaining 11 were normal healthy controls (7 males and 4 females, mean age: $13.9 \pm 6.6$ years old, age range: 5-21 years old). These individuals with ASD had the core symptoms of the DSM-5 diagnostic criteria for ASD without any abnormal neurological symptoms (e.g., seizure or movement disorders). The sex and age distributions were not significantly different between the two groups. The 20 individuals with ASD and the 11 individuals in the control group were matched on home environment, feeding habits and full intelligent quotient (IQ) scores (Table 1). The 11 normal controls were considered to be physically and mentally healthy based on initial physical and mental screening tests. At the initial screening, physical (resting blood pressure and heart rate) and clinical laboratory examinations (hematology and plasma chemistry, including plasma fatty acids, cholesterol and triglycerides) were performed on all 31 participants. These 31 participants did not have any abnormalities in their physical examinations and laboratory findings. The IQs of the individuals were estimated using the Wechsler Intelligence Scale for children and adolescents aged 6-16 years old [30] or the respective scale for adults (WAIS-R) (Table 1). Comorbid psychiatric disorders were evaluated based on the Structured Clinical Interview for DSM-IV Axis I Disorders (SCID). None of the ASD or control individuals had any history of neurological conditions, including seizure, movement disorders, head injury, Attention-Deficit Hyperactivity Disorder or learning disorders. The additional inclusion criteria were as follows: (a) the absence of any other medical or comorbid psychiatric disorders; (b) a baseline verbal or full IQ greater than 70 as calculated by the Wechsler Intelligence Scale for Children-Revised Manual (WAIS-R) [30] or the respective scale for adults [31] (WISC) because subjects with highfunctioning pervasive developmental disorders were required to have a total IQ of at least 70 [32]; and (c) no treatment with antidepressants, anxiolytic medications or neuroleptics within the three months prior to the study (the treatment of ADHD symptoms with stimulants was allowed during this study, provided that the patient's dosage was stable for at least 3 months before and during the study).

All procedures performed in this study were accordance with the ethical standards of the Fujimoto Medical Clinic in Kobe City, Japan (Medical doctor, Kunio Yui) with the 1964 Declaration of Helsinki and its later amendments or comparable ethical standards. This ethics committee is registered with Pharmaceuticals and Medical Devices Agency of Japan to register the IRB information (http://www.info. pmda.go.jp/). Most of the participants in this study were young people under the legal age of 20 years; thus, we obtained parental permission and applied information on the behalf of these individuals (e.g.,

Table 1. Subject characteristics and urinary levels of HEL, 8-OHdg and TAP, and the SRS scores in the 20 individuals with ASD and 11 normal controls.

\begin{tabular}{|c|c|c|c|c|}
\hline Variables & $\operatorname{ASD}(\mathbf{n}=\mathbf{2 0})$ & Controls $(\mathrm{n}=11)$ & $\mathbf{U}$ & $P$ values \\
\hline Age (Years) & $11.1 \pm 5.2$ & $13.9 \pm 6.6$ & 84,00 & 0.30 \\
\hline Sex (male/female) & $7 / 13$ & $4 / 7$ & $\chi^{2}=0.00$ & 1.00 \\
\hline Full IQ & $100.9 \pm 25.91$ & $109.38 \pm 18.45$ & 32.00 & 0.52 \\
\hline \multicolumn{5}{|c|}{ Scores of Autism Diagnostic Interview-Revised (ADI-R) } \\
\hline Domain A (social) & $13.6 \pm 6.3$ & - & & \\
\hline Domain B (communication) & $8.0 \pm 4.5$ & - & & \\
\hline Domain C (stereotyped) & $11.2 \pm 6.0$ & - & & \\
\hline \multicolumn{5}{|l|}{ Urinary levels } \\
\hline HEL (pmol/mL, Cre) & $77.10 \pm 30.56$ & $1.76 \pm 26.16$ & 53.00 & $0.018^{*}$ \\
\hline 8-OhdG (ng/mL Cre) & $11.31 \pm 5.50$ & $19.51 \pm 32.16$ & 105.50 & 0.86 \\
\hline TAP ( $\mu$ MUric Acid equivalents) & $2986.18 \pm 999.25$ & $4169.71 \pm 1285.03$ & 62.00 & $0.04 *$ \\
\hline Plasma SOD levels (U/ml) & $3.86 \pm 3.12$ & $3.64 \pm 2.82$ & 103.00 & 0.79 \\
\hline \multicolumn{5}{|l|}{ Subscale scores of the SRS } \\
\hline Irritability & $12.80 \pm 4.4$ & $12.73 \pm 3.70$ & 8.50 & $0.000 * *$ \\
\hline Social withdrawal & $25.80 \pm 7.13$ & $3.10 \pm 4.7$ & 40.00 & $0.000 * *$ \\
\hline Stereotypy & $41.25 \pm 11.05$ & $4.82 \pm 7.17$ & 1.50 & $0.000 * *$ \\
\hline Hyperactivity & $21.60 \pm 5.28$ & $4.73 \pm 5.49$ & 2.50 & $0.000^{* *}$ \\
\hline Inappropriate speech & $26.35 \pm 27.00$ & $2.18 \pm 3.97$ & 0.50 & $0.000 * *$ \\
\hline Total score & $114.94 \pm 31.34$ & $17.36 \pm 23.40$ & 3.00 & $0.000 * *$ \\
\hline
\end{tabular}

HEL, hexanolyl-lysinr; 8-OHdG, 8-hydoxy-2'-deoxyguanosine; TPA, Total Antioxidant Power; SRS, Social Responsiveness Scale; Values are mean \pm SD * $p<0.05, * * p<0.001$ compared to normal controls. 
Yui K (2018) The role of imbalance between urinary hexanoyl-lysine and total antioxidant capacity levels and its relation to plasma superoxide dismutase levels in autism spectrum disorder

recognizing whether each participant's urinary TAP and plasma SOD levels were within the standard values according to SRL, Inc, Tokyo, Japan). The standard values of TAP levels were within standard ranges established by the Department of Pediatrics, Tokyo Metropolitan Fuchu Medical Center for the Disabled, Tokyo, Japan (3700 - 4000 $\mu \mathrm{M}$ uric acid equivalents in 8 normal healthy subjects aged 6-37 years) [33], and those of plasma SOD levels were within standard ranges established by SRL, Inc., Tokyo, Japan (1.8-3.2 U/ml in 164 healthy subjects) [34]. The studies have been approved by the appropriate institutional and/or national research ethics committee and have been performed in accordance with the ethical standards as laid down in the 1964 Declaration of Helsinki and its later amendments or comparable ethical standards. Written informed consent was obtained from the participants and/or their parents. This study was registered at . or.jp/jmactr (the Clinical Trials Registry, Japan Medical Association, 2010/6/14; renewal,2015/12/31; ID: JMA-IIA00162).

\section{Assessment of dietary nutrient intake}

Urinary levels of TAC may be affected by dietary food intake may [26,27]. Berries, fruits, nuts, chocolate, vegetables [35], and cafe and soybean oil had the highest antioxidant values [36]. Thus, participants who habitually ingested foods and snacks containing high antioxidant values (berries, fruits, nuts, chocolate, vegetables, coffee and soybean oil) were excluded from the study.

To assess the daily food intake, a semi-constructive questionnaire for the Japanese (DHQ) was performed using the junior high school version (DHQ15) (DHQ Support Center, http://www.ebnjapan.org/). The DHQ15 consisted of 72 questions on the frequency of intake of 150 various food and beverage items and cooking methods. DHQ15 was administered one month before the study on randomly selected subsamples of 10 individuals with ASD and 10 normal controls by the order of their submission to medical consultation during January 2013 and June 2015. The food and beverage items and portion sizes in the questionnaire were derived primarily from the data in the Overview of Dietary Reference Intake for Japanese [37]. If there was any missing or unclear information recorded on the DHG 15 by the parents or subjects, the researcher (KY) questioned them by phone or e-mail, and information was corrected in the DHQ 15. The estimated intake of nutrients was calculated using a dedicated program for the DHQ system (DHQ Support Center, Tokyo, Japan) [38]. The validity of theDHQ15 has been already verified [39].

\section{Estimation of dietary TAC}

Urinary levels of TAC have been reported to be affected by dietary food intake. Grape juice (200 ml) [38] and cocoa powder (40 g) [39] increased urinary TAC. Thus, a dietary TAC value was assigned to each food item in the DHQ15. The 31 participants in this study were children or young adolescents with age range: 6-22 years old, their nutrients were less varied and were of smaller amounts than those of adults. We therefore calculated dietary TAC value of the remaining 34 foods in the DHQ15 according to a previous study [35,42]. TAC values were expressed as $\mathrm{mmol} \mathrm{Fe} \mathrm{F}^{2+} / 100 \mathrm{~g}$ of food for ferric. reducing ability of plasma (FRAP) [42] or mmol/100g oxygen radical absorbance capacity (ORAC) [35,42]. Finally, The TAC values of 18 foods were calculated as FRAP or those of 4 foods were calculated as ORAC.

\section{Urinary assay of HEL, 8-OHdG and TAC levels}

Urines were collected as a spot sample and immediately stored at-80C until analysis. After the dissolving process, the urines were centrifuged to remove all insoluble materials. The specialists at the Department of Pediatrics, Tokyo Metropolitan Fuchu Medical Center for the Disabled (Tokyo, Japan) measured the urinary levels of HEL, 8-OHdG and TAC.

The urinary levels of HEL were measured in duplicate using a competitive ELISA kit (Japan International Cooperation Agency-JICA, Shizuoka, Japan) [43].

For measurement of the urinary levels of $8-\mathrm{OHdG}$, urine samples were centrifuged and the supernatant after proper dilution was used in duplicate for assessment with a competitive enzyme-linked immunosorbent assay kit (8-OHdG check ELISA kit, JalCA, Japan Institute for the Control of Aging, Shizuoka, Japan). These results were then corrected to the urinary concentration of creatinine, and the urinary $8-\mathrm{OHdG} /$ creatinine levels were used in subsequent analyses [44].

For measurement of the urinary levels of urinary total antioxidant capacity, the urinary antioxidant capacity was determined by competitive enzyme-linked Immunosorbent assay (ELISA) [44]. This assay provides accurate determination of the total antioxidant activity in a sample that results from the combined activities of the constituents (Oxford Biomedical Research).

\section{Plasma levels of SOD}

Plasma SOD levels were estimated from the rate of decrease in nitrite produced by hydroxylamine and the superoxide anions based on the nitrite method, using a Versa max instrument (Molecular Devices Co, Tokyo, Japan). Human plasma was assayed using an SOD Assay Kit (Takara Bio, Tokyo) according to the cytochrome $\mathrm{c}$ method, and the plasma SOD levels are expressed as units per milliliter. The assay sensitivity was $0.3 \mathrm{U} / \mathrm{ml}$. The intra-assay and inter-assay coefficients were 2.11 and $2.10 \mathrm{U} / \mathrm{ml}$, respectively.

\section{Assessment of social impairment}

The social responsiveness scale (SRS) can be used to distinguish clinically significant ASD from other psychiatric disorders based on varying levels of social impairment [45]. The SRS is a 65-item questionnaire completed by subjects' parents for a quantitative assessment of autistic traits across a wide spectrum of children aged 4-18 or 19 and more years (adult version). The total SRS raw scores range from 0 to 195 , with the highest scores corresponding to significant social impairment, as observed in individuals with severe ASD [47]. The SRS can reliably distinguish other psychiatric conditions from ASD [46] and assess the severity of autistic trait [47].

\section{Statistical analyses}

Because the data were not normally distributed, the non-parametric Mann-Whitney U test for multiple comparisons was used to determine the significant differences in the urine levels of HEL, 8-OHdG and TAC and plasma SOD levels as well as the five subscales and total scores on the SRS, and the intake of nutrients between the random subsamples of 10 individuals with ASD and 10 of the normal controls. Moreover, stepwise regression analysis was used to confirm the relationship between the urinary oxidative stress-related biomarkers (levels of HEL, 8-OHdG and TAC) and plasma SOD levels, and the other main variables, adjusting for the two subject groups, and the SRS five subscale and total scores (Table 2). We conducted statistical analyses using SPSS version 18.0 (IBM Tokyo, 2009). 
Yui K (2018) The role of imbalance between urinary hexanoyl-lysine and total antioxidant capacity levels and its relation to plasma superoxide dismutase levels in autism spectrum disorder

Table 2. Results of the stepwise regression analysis.

\begin{tabular}{|c|c|c|c|c|c|}
\hline Model & Model & & Model coefficients & & \\
\hline & $\mathbf{R}^{2}$ & p-value & B & Beta & p-value \\
\hline $\begin{array}{c}\text { TAP } \\
\text { Step } 1\end{array}$ & 0.219 & $0.008 * *$ & & & \\
\hline Group $(1=\mathrm{ASD}, 2=$ controls $)$ & & & $1183.529 \pm 415.231$ & 0.468 & $0.008 * *$ \\
\hline Step 2 & 0.416 & & & & \\
\hline Group $(1=\mathrm{ASD}, 2=$ controls $)$ & & & $109.753 \pm 725.554$ & 1.229 & $0.000 * *$ \\
\hline SRS cognition scores & & & $86.149 \pm 28.034$ & 0.881 & $0.005^{* *}$ \\
\hline $\begin{array}{c}\text { HEL } \\
\text { Step } 1\end{array}$ & 0.212 & $0.009 * *$ & & & \\
\hline SRS awareness scores & & $0.009 * *$ & $2.244 \pm 0.803$ & 0.460 & $0.009 * *$ \\
\hline
\end{tabular}

$\mathrm{TAP}=$ total antioxidant power; $\mathrm{R}^{2}=\mathrm{R}$-squared values; $\mathrm{B}=$ unstandardized coefficients; $\mathrm{ASD}=$ autism spectrum disorder; $\mathrm{SRS}=$ Social Responsiveness $\mathrm{Scale} ; * p<0.05, * * p<0.001$, significant contribution.

\section{Results}

\section{Study population}

Clinical features of in the 20 individuals with ASD were characterized by failure of normal back-and-forth conversation ( $n$ $=8)$, deficits in socio-emotional reciprocity $(n=9)$ and deficits in understanding relationships $(\mathrm{n}=3)$. The mean total SRS score for our patients was $111.0 \pm 33.1$ (Table 1). Previous studies reported total SRS scores of 120.21 for children and adolescents with moderate-tosevere ASD [48]. Considering that an SRS total score between 60 and 80 is associated with a mild form of ASD [49], our patients suffered moderate or severe social impairment from ASD. Ages did not differ significantly between the ASD and control groups $(p=0.30)$.

\section{Urinary levels of oxidative stress markers}

There was a significant increase in urinary HEL levels $(p=0.018)$ and a significant decrease in urinary TAC levels $(p=0.049)$ in the 20 individuals with ASD compared to those observed in the 11 normal controls. Urinary levels of 8-OHdG and plasma levels of SOD were not significantly different between the groups (Table 1).

\section{Predictor variables}

Table 2 summarizes the results of our stepwise multiple regression analysis. Urinary levels of TAC contributed significantly to the variables after adjusting for the two subject groups, urinary HEL levels, 8-OHdG levels, plasma SOD levels, and the five subscale and total ABC scores (Step 1, $\mathrm{R}^{2}=0.216, \mathrm{P}=0.008$; step 2, $\mathrm{R}^{2}=0.411, p=0.001$ ). Using Group used as a dependent variable showed a significance of contribution to the urinary TAC levels (step 1, unstandardized coefficients, $B=1152.564 \pm 407.338, \beta=0.5465$, and $p=0.008$; step 2, unstandardized coefficients, $B=3027.688 \pm 713.591, \beta=1.222$, and $p=$ 0.000 ) and to the SRS cognition scores (unstandardized coefficients, $B$ $=83.864 \pm 27.572, \beta=0.876$, and $p=0.005$ ).

Furthermore, the procedure using urinary HEL levels as the independent variable terminated at step 2, explaining $22.1 \%$ of the variance. The SRS subscale and total scores being used as the dependent variables, the statistical significance of contribution of the SRS subscale of awareness to the urinary HL levels (unstandardized coefficients, $B=2.312 \pm 0.807, \beta=0.470$, and $p=0.008$ ). These findings indicate that urinary levels of TAC and HEL were predictable measures for differences in the biomarkers and the SRS scores between the ASD group and the control group (Table 2).

\section{Association between urinary HEL, TAC and 8-OHdG levels, and plasma SOD levels and their relations to the SRS scores}

There were significant correlations between urinary TAC levels, and plasma SOD levels $(r=0.46, p=0.04)$ and SRS subscale scores of cognition $(\mathrm{r}=0.47 . p=0.04)$ in the ASD group. Urinary HEL levels were significantly correlated with the SRS subscales of awareness $(r=0.60$. $p=0.005)$ and mannerisms $(r=0.69, p=0.001)$. Plasma SOD levels significantly correlated with SRS subscale scores of communications $(r$ $=0.58, p=0.007)$ and total scores $(r=0.65, p=0.02)$.

\section{Assessment of Nutrient Intake}

There were no significant differences in weight, high, energy intake, or in the intake of protein $(p=1.00)$, cholesterol $(p=0.49)$, vitamin B2 $(p=0.97)$, vitamin B12 $(p=0.97)$, vitamin C $(p=0.18)$, omega-6 $(p=$ $0.44)$, iron $\mathrm{P}=0.55)$, copper $(p=0.55)$, omega-3 ( $p=0.50)$ and omega- 6 PUFAs $(p=0.44)$ between the ASD and control groups (Table 3 ).

\section{Dietary total antioxidant power}

The ASD group consumed significantly more dietary TAC in the form of chocolate $(p=0.02)$, cookies and biscuits

$(p=0.04)$, and jam and marmalade $(p=0.007)$ compared to the control group. However, there was no significant correlation between dietary TAC and urinary levels of TAP $(\mathrm{r}=0.02-0.57, p=0.95-0.053)$ in ASD groups (Table 4).

\section{Discussion}

The present study first measured a set of urinary levels of HEL, TAC and 8-OHdG, and revealed significantly decreased levels of TAC and significantly increased levels of HEL in urine of the 20 individuals with ASD compared with the 11 healthy controls. It was also shown that there were no significant differences in urinary 8-OHdG levels between the groups.

Stepwise multiple regression analysis identified significant correlations between the urinary levels of both TAC and HEL and the adjusting variables (the two subject groups, the levels of other urinary biomarkers and the total ABC scores) in the whole population. Thus, the urinary levels of TAC and HEL were appropriate models for distinguishing the ASD group from the control group and significantly predicted the adjusting variables. These findings suggest that the urinary levels of TAC and HEL may contribute to social impairment in individuals with ASD.

A previous study revealed that the urinary levels of TAC were significantly lower without a concomitant increase in urinary catalase 
Yui K (2018) The role of imbalance between urinary hexanoyl-lysine and total antioxidant capacity levels and its relation to plasma superoxide dismutase levels in autism spectrum disorder

Table 3. The intake of nutrients in the random subsamples of 10 individuals with ASD and random ubsample of 10 normal controls.

\begin{tabular}{|c|c|c|c|c|}
\hline & $\operatorname{ASD}(n=10)$ & Control $(n=10)$ & $\mathbf{U}$ & $p$ value \\
\hline Age (years) & $11.4 \pm 3.9$ & $10.1 \pm 3.9$ & 35 & 0.45 \\
\hline $\operatorname{High}(\mathrm{cm})$ & $142.1 \pm 21.7$ & $141.1 \pm 20.2$ & 42.5 & 0.84 \\
\hline Weight (kg & $39.5 \pm 21.6$ & $39.2 \pm 18.1$ & 45.5 & 1.00 \\
\hline Energy (kcal) & $2166.0 \pm 523.6$ & $2231.8 \pm 617.5$ & 40.0 & 0.72 \\
\hline Fat (g/day) & $71.6 \pm 26.2$ & $78.8 \pm 23.3$ & 38.0 & 0.60 \\
\hline Protein (g/day) & $79.2 \pm 23.3$ & $80.8 \pm 27.0$ & 43.0 & 0.91 \\
\hline Animal protein (mg/day) & $32.4 \pm 8.0$ & $33.8 \pm 12.0$ & 41.0 & 0.78 \\
\hline Cholesterol (mg/day) & $138.4 \pm 179.0$ & $127.8 \pm 134.6$ & 37.0 & 0.55 \\
\hline Carbohydrates (g/day) & $297.8 \pm 58.6$ & $292.2 \pm 86.8$ & 45.0 & 1.00 \\
\hline Vitamin B2 (mg/day) & $1.8 \pm 0.71$ & $7 \pm 0.8$ & 44.0 & 0.97 \\
\hline Vitamin B6 (mg/day) & $1.4 \pm 0.5$ & $1.3 \pm 0.4$ & 42.5 & 0.84 \\
\hline Vitamin B12 (mg/day) & $7.3 \pm 4.0$ & $7.0 \pm 2.3$ & 44.5 & 0.97 \\
\hline Vitamin C (mg/day) & $1678.0 \pm 79.4$ & $119.4 \pm 74.5$ & 28.0 & 0.18 \\
\hline Iron (mg/day) & $9.4 \pm 2.7$ & $8.3 \pm 3.2$ & 37.0 & 0.55 \\
\hline Copper (g/day) & $1.3 \pm 0.4$ & $1.2 \pm 0.4$ & 37.0 & 0.55 \\
\hline Unsaturated fatty acid (g/day) & $15.8 \pm 5.21$ & $6.7 \pm 7.0$ & 38.0 & 0.60 \\
\hline Saturated fatty acid (g/day) & $23.9 \pm 13.2$ & $25.8 \pm 10.0$ & 38.0 & 0.60 \\
\hline Omega-3 (mg/day) & $216.5 \pm 161.6$ & $192.7 \pm 90.8$ & 36.0 & 0.50 \\
\hline Omega-6 (mg/day) & $384.5 \pm 230.7$ & $357.7 \pm 121.3$ & 35.0 & 0.44 \\
\hline
\end{tabular}

Values are mean $\pm \mathrm{SD}$

Table 4. Dietary TAP in the random subsamples of 10 individuals with ASD and 10 normal controls.

\begin{tabular}{|c|c|c|c|c|}
\hline & $\operatorname{ASD}(n=10)$ & Control $(n=10)$ & $\mathbf{U}$ & $p$ value \\
\hline \multicolumn{5}{|l|}{ FRAP } \\
\hline \multicolumn{5}{|l|}{ Non-alcoholic beverages } \\
\hline Green tea & $7.13 \pm 5.7$ & $8.72 \pm 4.17$ & 42.5 & 0.58 \\
\hline Fruit juice & $2.79 \pm 4.28$ & $1.89 \pm 4.19$ & 31.5 & 0.17 \\
\hline \multicolumn{5}{|l|}{ Vegetables } \\
\hline Cabbage & $0.26 \pm 0.26$ & $0.16 \pm 0.13$ & 39.5 & 0.44 \\
\hline Radishes & $0.45 \pm 0.25$ & $0.31 \pm 0.26$ & 33.5 & 0.22 \\
\hline Tomatoes & $0.08 \pm 0.08$ & $0.10 \pm 0.09$ & 43 & 0.63 \\
\hline Lettuce & $0.09 \pm 0.05$ & $0.07 \pm 0.06$ & 41 & 0.53 \\
\hline Lotus root & $1.10 \pm 0.77$ & $0.80 \pm 0.44$ & 44.5 & 0.68 \\
\hline Carrot & $0.23 \pm 0.21$ & $0.17 \pm 0.10$ & 44.5 & 0.68 \\
\hline Other salted pickles & $0.002 \pm 0.006$ & $0.065 \pm 0.071$ & 8 & 0.21 \\
\hline Seevegetables & $0.57 \pm 0.49$ & $0.70 \pm 0.71$ & 27.5 & 0.09 \\
\hline \multicolumn{5}{|l|}{ Fruits } \\
\hline Oranges & $0.65 \pm 0.78$ & $0.41 \pm 0.51$ & 39.5 & 0.44 \\
\hline Strawberries & $1.10 \pm 1.78$ & $0.44 \pm 0.48$ & 43 & 0.63 \\
\hline \multicolumn{5}{|l|}{ Sugar confectioneries } \\
\hline Chocolate & $0.94 \pm 0.80$ & $0.29 \pm 0.33$ & 20 & $0.02 *$ \\
\hline Japanese sweets with a sweet filling & $0.02 \pm 0.03$ & $0.04 \pm 0.05$ & 49 & 0.97 \\
\hline Jam and marmalade & $0.15 \pm 0.13$ & $0.00 \pm 0.00$ & 45 & $0.007 *$ \\
\hline Cookies and biscuits & $0.06 \pm 0.05$ & $0.02 \pm 0.02$ & 22 & $0.04 *$ \\
\hline Pancakes & $0.03 \pm 0.03$ & $0.02 \pm 0.02$ & 0.53 & \\
\hline Japanese noodles & $0.39 \pm 0.30$ & $0.24 \pm 0.23$ & 33 & 0.22 \\
\hline \multicolumn{5}{|l|}{ ORAC } \\
\hline Breakfast cereals & $0.63 \pm 0.46$ & $0.42 \pm 0.35$ & 40.5 & 0.48 \\
\hline Egg & $0.03 \pm 0.01$ & $0.02 \pm 0.01$ & 35 & 0.28 \\
\hline Meat and meet products & $0.17 \pm 0.09$ & $0.20 \pm 0.20$ & 43.5 & 0.63 \\
\hline Poultry and poultry products & $0.06 \pm 0.05$ & $0.07 \pm 0.07$ & 42.5 & 0.58 \\
\hline
\end{tabular}

$\mathrm{FRAP}=$ ferric reducing ability of plasma; ORAC $=$ oxygen radical absorbance capacity, Values are mean $\pm \mathrm{SD} * p<0.05$

activity and total thiol molecules, which are the main indicators of antioxidant capacity [50] in 29 children with ASD aged 6-12 years than in 24 age-matched healthy controls [6]. Further, 34 adolescent individuals with Asperger syndrome (mean age $12.89 \pm 2.58$ years) showed significantly reduced plasma and erythrocyte levels of TAP compared to 34 age-matched controls, indicating a chronically low detoxifying capacity in these ASD individuals [25]. Regarding urinary HEL levels, a previous study reported that 24 children with ASD aged 5-12 years showed elevated levels of urinary HEL without concomitant alterations in the urinary levels of 8 -OHdG or the erythrocyte levels of SOD, which may result from a deficit in membrane fluidity [11]. Further, a case-control study reported that 68 children with ASD aged 3-10 years showed significantly increased levels of 8 -OHdG in peripheral lymphocyte DNA compared to 54 
Yui K (2018) The role of imbalance between urinary hexanoyl-lysine and total antioxidant capacity levels and its relation to plasma superoxide dismutase levels in autism spectrum disorder

age-matched healthy controls [10], indicating a deficit in antioxidant and methylation capacity that promote cellular damage and alter epigenetic gene expression. As indicated in this earlier works, prooxidant environmental stressors may act in conjunction with a genetic predisposition toward the development of ASD [10]. Previous studies evaluated the following parameters: 1) urinary levels of TAP alone [25]; 2) urinary levels of TAC in concomitance with urinary catalase activity and total thiol molecules [6];3) urinary levels of 8 -OHdG alone [10,51]; and 4) urinary levels of $8-\mathrm{OHdG}$ in concomitance with HEL [11]. According to recent measurements, a set of oxidative stressrelated biomarkers such as urinary levels of HEL, TAC and 8-OHdG may provide useful information on neuronal damage by oxidative stress [52,53]. The present study first examined the urinary levels of this set of three oxidative stress-related biomarkers in ASD.

The significant increase in urinary HEL levels and the reduction in urinary TAC levels in individuals with ASD demonstrated in this study indicated an imbalance between oxidative stress and antioxidant capacity. Moreover, stepwise regression analysis revealed that the urinary levels of TAC and HEL were reliable measures for distinguishing the ASD group from the control group. A previous review article suggested that declining ROS levels are coupled with increased antioxidant enzyme activities, depending on the formation of antioxidant enzymes relative to the changes in the levels of free radicals [54]. Moreover, growing evidence indicates that the imbalance between ROS production and TAC may correlate with ASD [55,56]. Thus, the present study first revealed detailed and concrete findings on the imbalance between oxidative stress and TAC in the urine of individuals with ASD. These findings may provide additional and useful information on the significance of imbalance between increased oxidative stress and impaired urinary antioxidant systems in the pathophysiology of individuals with ASD. Although elevated levels of 8-OHdG in plasma or urine is a commonly used biomarker for assessing oxidative DNA damage during inflammatory disease and pro-oxidant exposures [10], DNA methylation analysis of ASD reveals multiple dysregulated biological pathways [57]. Thus, the ASD group in this study may be a subset of ASD without DNA methylation damage.

Stepwise regression analysis and Spearman correlation coefficients revealed that urinary TAC levels were significantly correlated to plasma SOD levels and the SRS subscale scores for cognition. Previous studies reported significant decrease in plasma and urine SOD and TAC levels in 47 sarcoma patients [58] and significant increase in urine SOD and TAC levels after administration of L-carnitine in 12 healthy subjects [59], indicating concomitant changes of plasma and urine SOD and TAC, supporting our findings. A previous study reported a significant positive correlation between plasma TAC levels and cognitive function in 105 subjects with early onset-first episode psychosis [60], and an association between lowered plasma TAP levels and cognitive dysfunction in 138 older adults with mild cognitive dysfunction [61] and in 90 healthy care workers [62]. The Spearman correlation coefficient also revealed a significant association between plasma SOD levels and the SRS subscale of communication. A previous study reported significant correlations between blood levels of SOD and psychosocial functioning such as "relation to friends" and "taking initiative and self-sufficiency" in 51 subjects with bipolar depression [63]. Social interaction-mediated lifespan extension of SOD mutants has been found in human [64]. Thus, SOD may be significantly correlated to social behavior, which supports our present findings.

Stepwise regression analysis and Spearman correlation coefficients further revealed that the urinary HEL levels were significantly correlated to the SRS subscale scores for awareness and mannerisms. Of reference, the plasma levels of 3-nitrotyrosine (3-MT), which is an oxidative stress marker [65], have previously been associated with low scores in SRS subscales of awareness in 18 children with ASD [66]. Sulforaphane, which has therapeutic potential against oxidative stress, was shown to improve SRS scores by $17 \%$ [48]. Thus, oxidative stress biomarkers are associated with the SRS scores, which supports our findings.

A previous assessment of daily nutrient intake using DHQ 15 revealed no significant differences in the intake of fat, protein, vitamin B2, vitamin B6, vitamin B12, vitamin C, omega-6, or omega-3 PUFAs between random subsamples of 10 individuals with ASD and those of 10 healthy controls. With respect to the relationship between dietary intake and the urinary levels of TAP, intake of virgin olive oil [67] and tryptophan-enriched cereal [68] have been shown to increase urinary TAC. Conversely, refined potato starch resulted in decreased urinary levels of TAC [69]. Therefore, the effect of dietary intake on urinary TAC may be variable, and differences in dietary intake between the groups may not affect urinary TAC.

The dietary TAC of chocolate, biscuits and cookies, jam and marmalade were significantly higher in the ASD group than in the control group. Cocoa products such as chocolate are good sources of dietary antioxidants [70]. Cookies containing chocolate chips have been shown to possess the highest antioxidant capacity [71]. Biscuits including $5 \%$ cocoa increased antioxidant properties [72]. Jam and marmalade processing has been shown to increase antioxidant capacity [73], and bilberry jams have been revealed as a good source of antioxidant compounds [74,75]. Thus, chocolate, cookies, biscuits, jam and marmalade possess higher antioxidant power. However, urinary TAC levels in the ASD group were significantly lower than those in the control group, indicating that antioxidant capacity in the ASD group may be impaired.

Oxidative stress contributes to many pathological conditions. Aerobic organisms have integrated antioxidant systems, which include enzymatic and non-enzymatic antioxidants that are usually effective in blocking the harmful effects of ROS [76]. Such systems include the endogenous antioxidant defense system [77] and intrinsic antioxidant defenses [78]. Most previous studies on antioxidant capacity in individuals with ASD have suggested a vulnerability in antioxidant enzymes [24] or a chronically low detoxifying capacity [25]. These antioxidant defense systems may be a part of the endogenous antioxidant systems. Indeed, recent research on antioxidant networks has demonstrated that antioxidant enzymes such as SOD, glutathione peroxidase, and glutathione act as an antioxidant network within specific intracellular or extracellular components of the antioxidant system [79]. Further, a recent work has suggested that the autophagylysosomal activities of these antioxidant enzymes may serve an essential function in preventing neurodegenerative diseases by removing damaged as part of an essential cellular antioxidant pathway [80]. These considerations taking together suggested that the endogenous intrinsic antioxidant system may be impaired in individuals with ASD. However, the present study did not examine the specific neurobiological roles of urinary TAP and plasma SOD, and further studies will need to determine which specific components may be affected in ASD.

Compromised intrinsic antioxidant defense contribute to bloodbrain barrier (BBB) disruption through oxidative damage to cellular molecules [81]. When BBB disruption occurs, brain-specific proteins circulating inside the brain, are observed in the peripheral blood as an 
Yui K (2018) The role of imbalance between urinary hexanoyl-lysine and total antioxidant capacity levels and its relation to plasma superoxide dismutase levels in autism spectrum disorder

index for estimating the extent of the increase in BBB permeability and brain damage [81]. As described above, overproduction of ROS results in oxidative stress the imbalance between ROS and antioxidant capacity (referred to oxidative stress) became toxic to neurons, damaging DNA, proteins and lipids, inducing neurodegeneration [4] such as ASD [82]. Thus, the imbalance between the generation of ROS and TAC may disrupt $\mathrm{BBB}$ and be related to impaired social responsiveness through biochemical processes such as imbalance between increased urinary levels of HEL as oxidants and urinary levels of TAC as antioxidant in individuals with ASD.

This study has some limitations. Previous studies examined urinary oxidative stress biomarkers such as F2-isoprostanes and their association with the activity of plasma enzymatic antioxidants such as SOD and glutathione peroxidase [83,84]. In this study, the urinary levels of 2-isoprostanes and plasma levels of glutathione were not measured; however, we examined a useful and informative set of oxidative stressrelated biomarkers and discovered novel important information on impaired antioxidant capacity that was not revealed in previous studies $[6,11,25,85]$. Second, ASD is most prevalent in males, with a male to female ratio of 4 to 1 [86]. However, in this study, the ASD and control groups were age- and gender-matched. Finally, the small sample size affects our ability to generalize our results to the entire population of individuals with ASD.

In conclusion, the present study firstly found reduced levels of TAC and increased levels of HEL in urine in individuals with ASD with a lack of significant alterations in urinary 8-OHdG levels. Thus, the imbalance between the generation of ROS and TAC was impaired in individuals with ASD. In addition, as stepwise regression analysis revealed that urinary TAC levels showed preferentially contribute to the adjusting variables compared to urinary HEL levels, urinary TAC levels may be more appropriate model for distinguishing the ASD group from the control groups. Thus, endogenous antioxidant defense systems may be impaired in young individuals with ASD. Plasma SOD levels may be associated with urinary TAC levels and may be related to the impaired antioxidant capacity. These defects in the balance between oxidative stress and antioxidant capacity in urine may be related to the pathophysiology of social impairment in individuals with ASD.

\section{Contribution of authors}

All authors met the criteria for this journal's authorship credit in the author guide

Each author's name next to the appropriate category.

Study conception and design: Kunio Yui

Acquisition of data: George Kunio Yui

Analysis and interpretation of data: Naoyaki Tanuma

Drafting of manuscript: Kunio Yui, Hitomi Sasaki

Critical revision: Hitomi Sasaki

\section{Acknowledgements}

Authors thank each subject for providing urine sample

\section{Funding source}

This study was supported by a small research grant such as Grantin-Aid for Scientific Research (C) (2014-2016) from the Ministry of Education, Culture, Sports, Science and Technology, Japan.

\section{Conflict of interest}

The authors are not currently applying for any patents relating to the content of the manuscript, and did not receive reimbursements, fees, funding, or salary from an organization that holds or has applied for patents relating to the content of the manuscript.

The authors declare that they have no conflict of financial and nonfinancial interest.

\section{Reference}

1. Hansen SN1, Schendel DE2, Parner ET1 (2015) Explaining the increase in the prevalence of autism spectrum disorders: the proportion attributable to changes in reporting practices. JAMA Pediatr 169: 56-62. [Crossref]

2. Picardi A, Fagnani C, Medda E, Toccaceli V, Brambilla P et al (2015) Genetic and environmental influences underlying the relationship between autistic traits and temperament and character dimensions in adulthood. Compr Psychiatry 58: 178-188. [Crossref]

3. Banerjee A, Luong JA, Ho A, Saib AO, Ploski JE (2016) Overexpression of Homer1 in the basal and lateral amygdala impairs fear conditioning and induces an autism-like social impairment. Mol Autism 7: 16. [Crossref]

4. Birben E1, Sahiner UM, Sackesen C, Erzurum S, Kalayci O (2012) Oxidative stress and antioxidant defense. World Allergy Organ J 5: 9-19. [Crossref]

5. Lambeth JD1 (2007) Nox enzymes, ROS, and chronic disease: an example of antagonistic pleiotropy. Free Radic Biol Med 43: 332-347. [Crossref]

6. Ranjbar A, Rashedi V, Rezaei M (2014) Comparison of urinary oxidative biomarkers in Iranian children with autism. Res Dev Disabil 5: 2751-2755.

7. Kern JK1, Jones AM (2006) Evidence of toxicity, oxidative stress, and neuronal insult in autism. J Toxicol Environ Health B Crit Rev 9: 485-499. [Crossref]

8. Bartosz G1 (2010) Non-enzymatic antioxidant capacity assays: Limitations of use in biomedicine. Free Radic Res 44: 711-720. [Crossref]

9. Eroglu M1, Sahin S, Durukan B, Ozakpinar OB, Erdinc N et al. (2014) Blood serum and seminal plasma selenium, total antioxidant capacity and coenzyme q10 levels in relation to semen parameters in men with idiopathic infertility. Biol Trace Elem Res 159: 46-51. [Crossref]

10. Melnyk S, Fuchs GJ, Schulz E, Kahler SG, Fussell JJ et al. (2012) Metabolic imbalance associated with methylation dysregulation and oxidative damage in children with autism. J Autism Dev Disord 42: 367-377. [Crossref]

11. Ghezzo A1, Visconti P, Abruzzo PM, Bolotta A, Ferreri C, et al. (2013) Oxidative Stress and Erythrocyte Membrane Alterations in Children with Autism: Correlation with Clinical Features. PLoS One 8: e66418. [Crossref]

12. Tokuda F, Sando Y, Matsui H, Yokoyama TN (2009) N epsilon- (hexanoyl) lysine, a new oxidative stress marker, is increased in metabolic syndrome, but not in obstructive sleep apnea. Am J Med Sci 338: 127-133. [Crossref]

13. Koca Y1, Ozdal OL, Celik M, Unal S, Balaban N (2003) Antioxidant activity of seminal plasma in fertile and infertile men. Arch Androl 49: 355-359. [Crossref]

14. Yavuzer H, Yavuzer S, Cengiz M, Erman H, Doventas A et al. (2016) Biomarkers of lipid peroxidation related to hypertension in aging. Hypertens Res 39: 42- 48. [Crossref]

15. Hung TH, Lo LM, Chiu TH, Li MJ, Yeh YL et al. (2010) A longitudinal study of oxidative stress and antioxidant status in women with uncomplicated pregnancies throughout gestation. Reprod Sci 7: 401-409. [Crossref]

16. Flynn JM1, Melov S (2013) SOD2 in mitochondrial dysfunction and neurodegeneration. Free Radic Biol Med 62: 4-12. [Crossref]

17. Wang L, Jia J, Zhang J, Li K (2016) Serum levels of SOD and risk of autism spectrum disorder: A case-control study. Int J Dev Neurosci 51: 12-16. [Crossref]

18. Yorbik O, Sayal A, Akay C, Akbiyik DI, Sohmen T (2002) Investigation of antioxidant enzymes in children with autistic disorder. Prostaglandins, Leukotrienes and Essential Fatty Acids 67: 341-343.

19. Sögüt S, Zoroglu SS, Özyurt H, Yilmaz HR, Ozugurlu F et al. (2003) Changes in nitric oxide levels and antioxidant enzyme activities may hay a role in the pathophysiolgical mechanisms involved in autism. Clin Chim Acta 33: 111-117. [Crossref]

20. Zoroglu SS, Armutcu F, Ozen S, Gurel A, Sivasli EO et al. (2004) Increased oxidative stress and altered activities of erythrocyte free radical scavenging enzymes in autism. Eur Arch Psychiatry Clin Neurosci 254: 143-147. [Crossref] 
Yui K (2018) The role of imbalance between urinary hexanoyl-lysine and total antioxidant capacity levels and its relation to plasma superoxide dismutase levels in autism spectrum disorder

21. Chauhan A, Chauhan V, Brown WT, Cohen I (2004) Oxidative stress in autism: increased lipid peroxidation and reduced serum levels of ceruloplasmin and transferrinthe antioxidant proteins. Life Sci 75: 2539-2549. [Crossref]

22. Tórsdóttir G, Hreidarsson S, Kristinsson J, Snaedal J, Jóhannesson Y (2005) Ceruloplasmin, superoxide dismutase and copper in autistic patients. Basic Clin Pharmacol Toxicol 96: 146-148. [Crossref]

23. Al-Gadani A, El-Ansary A, Attas O, Al-Ayadhi L (2009) Metabolic biomarkers related to oxidative stress and antioxidant status in Saudi autistic children. Clin Biochem 42: 1032-1040. [Crossref]

24. Meguid NA1, Dardir AA, Abdel-Raouf ER, Hashish A (2011) Evaluation of oxidative stress in autism: defective antioxidant enzymes and increased lipid peroxidation. Biol Trace Elem Res 143: 58-65. [Crossref]

25. Parellada M1, Moreno C, Mac-Dowell K, Leza JC, Giraldez M et al. (2012) Plasma antioxidant capacity is reduced in Asperger syndrome. J Psychiatr Res 46: 394-401. [Crossref]

26. González-Flores D, Gamero E, Garrido M, Ramírez R, Moreno D et al. (2012) Urinary 6-sulfatoxymelatonin and total antioxidant capacity increase after the intake of a grape juice cv. Tempranillo stabilized with HHP. Food Funct 3: 34-39. [Crossref]

27. Scholl TO, Leskiw M, Chen X, Sims M, Stein TP (2005) Oxidative stress, diet, and the etiology of preeclampsia. Am J Clin Nutr 81: 1390-1396. [Crossref]

28. American Psychiatric Association (2013) Diagnostic and statistical manual of mental disorders. 5th ed. Washington, DC. American Psychiatric association.

29. Rutter M, Le Couteur A, Lord C (2003) ADI-R Autism Diagnostic Interview Revised. Manual. Los Angeles Western Psychological Services.

30. Wechsler D (1974) Wechsler Intelligence Scale for Children-Revised Manual. New York, The Psychological Corporation.

31. Wechsler D (1981) Wechsler Intelligence Scale for Children-Revised Manual. San Antonio, TX. The Psychological Corporation.

32. Koyama T, Kamio Y, Inada N, Kurita H (2009) Sex differences in WISC-III profiles of children with high-functioning pervasive developmental disorders. $J$ Autism Dev Disord 39: 135-141. [Crossref]

33. Miyata R, Tanuma N, Sakuma H, Hayashi H (2016) Circadian Rhythms of Oxidative Stress Markers and Melatonin Metabolite in Patients with Xeroderma Pigmentosum Group A. Oxid Med Cell Longev. [Crossref]

34. Komatsu F, Kagawa Y, Sakuma M, Kawabatabe T, Kaneko Y et al. (2006) Investigation of oxidative stress and dietary habits in Mongolian people, compared to Japanese people. Nutr Metab (Lond) 7: 3-21. [Crossref]

35. Carlsen MH, Halvorsen BL, Holte K, Bøhn SK, Dragland S et al. (2010) The total antioxidant content of more than 3100 foods, beverages, spices, herbs and supplements used worldwide. Nutr J 9: 3. [Crossref]

36. Pellegrini N, Serafini M, Colombi B, Del Rio D, Salvatore S et al. (2003) Total antioxidant capacity of plant foods, beverages and oils consumed in Italy assessed by three different in vitro assays. J Nutr 133: 2812-2819. [Crossref]

37. Ministry of Health, Labour, and Welfare, Overview of Dietary Reference Intake for Japanese (2015) Ministry of Health, Labour, and Welfare, Tokyo.

38. Kobayashi Y, Hattori M, Wada S, Iwase H, Kadono M et al. (2013) Assessment of daily food and nutrient intake in Japanese type 2 diabetes mellitus patients using dietary reference intakes. Nutrients 5: 2276-2288. [Crossref]

39. Okuda M, Sasaki S, Bando N, Hashimoto M, Kunitsugu I et al. (2009) Carotenoid, tocopherol, and fatty acid biomarkers and dietary intake estimated by using a brief selfadministered diet history questionnaire for older Japanese children and adolescents. $J$ Nutr Sci Vitaminol (Tokyo) 55: 231-241. [Crossref]

40. González-Flores D, Gamero E, Garrido M, Ramírez R, Moreno D et al. (2012) Urinary 6-sulfatoxymelatonin and total antioxidant capacity increase after the intake of a grape juice cv. Tempranillo stabilized with HHP. Food \& Funct 3: 34-39. [Crossref]

41. Roura E, Almajano MP, Bilbao ML, Andrés-Lacueva C, Estruch R et al. (2007) Human urine: epicatechin metabolites and antioxidant activity after cocoa beverage intake. Free Rad Res 41: 943-949. [Crossref]

42. Kobayashi S, Murakami K, Sasaki S, Uenish K, Yamasaki M et al. (2012) Dietary total antioxidant capacity from different assays in relation to serum C-reactive protein among young Japanese women. Nutr J 11: 91. [Crossref]

43. MatsuI Y, Satoh K, Miyazaki T, Shirabe S, Atarashi R et al. (2011) High sensitivity of an ELISA kit for detection of the gamma-isoform of 14-3-3 proteins: usefulness in laboratory diagnosis of human prion disease. BMC Neurol 11: 120. [Crossref]
44. Boonla C, Wunsuwan R, Tungsanga K, Tosukhowong P (2007) Urinary 8-hydroxydeoxyguanosine is elevated in patients with nephrolithiasis. Urol Res 35: 185-191. [Crossref]

45. Wang J, Lee LC, Chen YS, Hsu JW (2012) Assessing autistic traits in a Taiwan preschool population: cross-cultural validation of the Social Responsiveness Scale (SRS). J Autism Dev Disord 42: 2450-2459. [Crossref]

46. Constantino JN1, Przybeck T, Friesen D, Todd RD (2000) Reciprocal social behavior in children with and without pervasive developmental disorders. J Dev Behav Pediatr 21: 2-11. [Crossref]

47. Di Martino A, Shehzad Z, Kelly C, Roy AK, Gee DG et al. (2009) Relationship between cingulo-insular functional connectivity and autistic traits in neurotypical adults. $\mathrm{Am} \mathrm{J}$ Psychiatry 166: 891-899. [Crossref]

48. Singh K, Connors SL, Macklin EA, Smith KD, Fahey JW (2014) Sulforaphane treatment of autism spectrum disorder (ASD). Proc Natl Acad Sci U S A 111: 1555015555. [Crossref]

49. Constantino JN, Davis SA, Todd Rd, Schindler MK, Gross MM et al. (2003) Validation of a brief quantitative measure of autistic traits: comparison of the social responsiveness scale with the autism diagnostic interview-revised. J Autism Dev Disord 33: 427- 433. [Crossref]

50. Balcerczyk A, Bartosz G (2003) Thiols are main determinants of total antioxidan capacity of cellular homogenates. Free Rad Res 37: 537-541. [Crossref]

51. Ming X, Stein TP, Brimacombe M, Johnson WG, Lambert GH et al. (2005) Increased excretion of a lipid peroxidation biomarker in autism. Prostaglandins Leukot Essent Fatty Acids 73: 379-384. [Crossref]

52. Collins AR1 (2005) Assays for oxidative stress and antioxidant status: applications to research into the biological effectiveness of polyphenols. Am J Clin Nutr 81: 261S-267S. [Crossref]

53. Song F, Jia W, Yao Y, Hu Y, Lei L (2007) Oxidative stress, antioxidant status and DNA damage in patients with impaired glucose regulation and newly diagnosed Type 2 diabetes. Clin Sci (Lond) 112: 599-606. [Crossref]

54. Ahmed RG (2005) Is there a balance between oxidative stress and antioxidant defense system during development? Med J Islamic World Acad Sci 15: 55-63.

55. James SJ, Rose S, Melnyk S, Jernigan S, Blossom S et al. (2009) Cellular and mitochondrial glutathione redox imbalance in lymphoblastoid cells derived from children with autism. FASEB J 23: 2374-2383. [Crossref]

56. Ghanizadeh A, Akhondzadeh S, Hormozi M, Makarem A, Abotorabi-Zarchi M et al. (2012) Glutathione-related factors and oxidative stress in autism, a review. Curr Med Chem 19: 4000-4005. [Crossref]

57. Nardone S, Sams DS, Reuveni E, Getselter D, Oron O et al. (2014) DNA methylation analysis of the autistic brain reveals multiple dysregulated biological pathways. Trans Psychiatry 4: e433. [Crossref]

58. Nathan FM, Singh VA, Dhanoa A, Palanisamy UD (2011) Oxidative stress and antioxidant status in primary bone and soft tissue sarcoma. BMC Cancer 11: 382 .

59. Yu C, Chuan-ji H, Chen-jing C, Peng-li L, Le-xin W et al. (2013) Urinary excretion of L-carnitine, acetyl-L-carnitine, propionyl-L-carnitine and their antioxidant activities after single dose administration of L-carnitine in healthy subjects. Braz. J. Pharm. Sci 49: $185-192$.

60. Martínez-Cengotitabengoa M, Micó JA, Arango C, Castro-Fomieles J, Graell M et al (2014) Basal low antioxidant capacity correlates with cognitive deficits in early onset psychosis. A 2-year follow-up study. Schizophr Res 156: 23-29. [Crossref]

61. Yuan L, Liu J, Ma W, Dong L, Wang W, Che R, Xiao R (2016) Dietary pattern and antioxidants in plasma and erythrocyte in patients with mild cognitive impairment from China. Nutrition 32: 193-198. [Crossref]

62. Özdemir PG1, Selvi Y, Özkol H, Aydın A, Tülüce Y et al. (2013) The influence of shift work on cognitive functions and oxidative stress. Psychiatry Res 210: 1219-1225. [Crossref]

63. Aydemir Ö., Çubukçuoglu Z, Erdin S, Tas C, Onur E et.al (2013) Oxidative stress markers, cognitive functions, and psychosocial functioning in bipolar disorder: an empirical cross-sectional study. Rev Bras Psiquiatr 36: 293-297. [Crossref]

64. Ruan H, Wu CW (2008) Social interaction-mediated lifespan extension of Drosophila $\mathrm{Cu} / \mathrm{Zn}$ superoxide dismutase mutants. Proc Natl Acad Sci U S A 105: 7506-7510. [Crossref]

65. Darwish RS, N. Amiridze, Arabi B (2007) Nitrotyrosine as an oxidative stress marker: evidence for involvement in neurologic outcome in human traumatic brain injury. $J$ Trauma 63: 439- 442. [Crossref] 
Yui K (2018) The role of imbalance between urinary hexanoyl-lysine and total antioxidant capacity levels and its relation to plasma superoxide dismutase levels in autism spectrum disorder

66. Frye RE, Delatorre R, Taylor H, Slattery J, Melnyk S et al. (2013) Redox metabolism abnormalities in autistic children associated with mitochondrial disease. Trans Psychiatry 3: e273. [Crossref]

67. Garrido M, González-Flores D, Marchena AM, Prior E, García-Parra J et al. (2013) A lycopene-enriched virgin olive oil enhances antioxidant status in humans. J Sci Food Agric 93: 1820-1826. [Crossref]

68. Bravo R, Matito S, Cubero J, Paredes SD, Franco L et al. (2013) Tryptophan-enriched cereal intake improves nocturnal sleep, melatonin, serotonin, and total antioxidan capacity levels and mood in elderly humans. Age (Dordr) 35: 1277-1285. [Crossref]

69. Vinson JA1, Demkosky CA, Navarre DA, Smyda MA (2012) High-antioxidant potatoes: acute in vivo antioxidant source and hypotensive agent in humans after supplementation to hypertensive subjects. J Agric Food Chem 60: 6749-6754. [Crossref]

70. Todorovic V, Redovnikovic IR, Todorovic Z, Jankovic G, Dodevska M et al. (2015) Polyphenols, methylxanthines, and antioxidant capacity of chocolates produced in Serbia. J Food Compost Anal 41: 137-143.

71. Sun Y, Hayakawa S, Ogawa M, Fukada K, Izumori K (2008) Influence of a rare sugar, d-Psicose, on the physicochemical and functional properties of an aerated food system containing egg albumen. J Agric Food Chem 56: 4789- 4796. [Crossref]

72. Ajibo GF, Oyerinde VO, Adeniyan OS (2015) Physiochemical and antioxidant properties of whole-wheat biscuits incorporated with Moringa oleifera leaves and cocoa powder. J Sci Res Rep 7: 195-206.

73. Kamiloglu S1, Pasli AA2, Ozcelik B2, Van Camp J3, Capanoglu E4 (2015) Influence of different processing and storage conditions on in vitro bioaccessibility of polyphenols in black carrot jams and marmalades. Food Chem 186: 74-82. [Crossref]

74. Poiana MA, Alexa E, Mateescu C (2012) Tracking antioxidant properties and color changes in low-sugar bilberry jam as effect of processing, storage and pectin concentration. Chem Cent J 6: 4. [Crossref]

75. Poiana MA, Munteanu MF, Bordean DM, Gligor R, Alexa E (2013) Assessing the effects of different pectins addition on color quality and antioxidant properties of blackberry jam. Chem Cent J 7:121. [Crossref]

76. Bhattacharyya A, Chattopadhyay R, Mitra S, Crowe SE (2014) Oxidative stress: an essential factor in the pathogenesis of gastrointestinal mucosal diseases. Physiol Rev 94: 329-354. [Crossref]
77. Miglio C, Pelus I, Raguzzini A, Villaño DV, Cesqui E et al. (2014) Fruit juice drinks prevent endogenous antioxidant response to high-fat meal ingestion. Br J Nutr 111: 294-300. [Crossref]

78. Papadia S, Soriano FX, Léveillé F, Martel MA, Dakin KA et al. (2008) Synaptic NMDA receptor activity boosts intrinsic antioxidant defenses. Nat Neurosci 11: 476487. [Crossref]

79. Liu S, Li X, Wu S, He J, Pang C et al. (2014) Fungal pretreatment by Phanerochaete chrysosporium for enhancement of biogas production from corn stover silage. Appl Biochem Biotechnol 174: 1907-1918. [Crossref]

80. Giordano S, Darley-Usmar V, Zhang J (2013) Autophagy as an essential cellular antioxidant pathway in neurodegenerative disease. Redox Biol 2: 82-90. [Crossref]

81. Hee-Tae Roh, Su-Youn Cho, Wi-Young S (2016) Obesity promotes oxidative stress and exacerbates blood-brain barrier disruption after high-intensity exercise. $J$ Sport Health Sci 1-6.

82. Kern JK, Geier DA, Sykes LK, Geier MR (2013) Evidence of neurodegeneration in autism spectrum disorder. Transl Neurodegener 2: 17. [Crossref]

83. Matayatsuk C, Lee CY, Kalpravidh RW, Sirankapracha P, Wilaira P et al. (2007) Elevated F2-isoprostanes in thalassemic patients. Free Radic Biol Med 43: 1649-1655. [Crossref]

84. Soto-Méndez MJ1, Aguilera CM2,3, Mesa MD et al. (2016) Strong Associations Exist among Oxidative Stress and Antioxidant Biomarkers in the Circulating, Cellular and Urinary Anatomical Compartments in Guatemalan Children from the Western Highlands. PLoS One 11: e0146921. [Crossref]

85. Ghanizadeh A1, Akhondzadeh S, Hormozi M, Makarem A, Abotorabi-Zarchi M et al (2012) Glutathione-related factors and oxidative stress in autism, a review. Curr Med Chem 19: 4000-4005. [Crossref]

86. Baron-Cohen S, Lombardo MV, Auyeung B, Ashwin E, Chakrabarti B et al. (2012) Why are autism spectrum conditions more prevalent in males? PLoS Biol 9: e100108. [Crossref]

Copyright: (C2018 Yui K. This is an open-access article distributed under the terms of the Creative Commons Attribution License, which permits unrestricted use, distribution, and reproduction in any medium, provided the original author and source are credited. 